\title{
Ictiofauna de sistemas cárstico-palustres con impacto antrópico: los petenes de Campeche, México
}

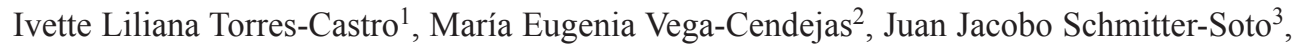 \\ Gerardo Palacio-Aponte ${ }^{4,5}$ \& Rocío Rodiles-Hernández ${ }^{6}$
}

1. El Colegio de la Frontera Sur, Unidad Campeche. Calle 10 x 61 \# 264, 24000 Campeche, Campeche, México; lilytorres90@hotmail.com

2. Centro de Investigación y Estudios Avanzados del IPN, Unidad Mérida. A.P. 73 "Cordemex”, 97310 Mérida, Yucatán, México; maruvega@mda.cinvestav.mx

3. El Colegio de la Frontera Sur, Unidad Chetumal. A.P. 424,77000 Chetumal, Quintana Roo, México; jschmitt@ecosur.mx

4. Centro EPOMEX, Universidad Autónoma de Campeche. Av. Agustín Melgar y Juan de Barrera s/n. 24030 Campeche, Campeche, México; gpalaciomx@gmail.com

5. Coordinación de Ciencias Sociales y Humanidades, Universidad Autónoma de San Luis Potosí. Av. Industrias 101-A, 78494 San Luis Potosí, San Luis Potosí, México; gpalaciomx@gmail.com

6. El Colegio de la Frontera Sur, Unidad San Cristóbal, A.P. 63, San Cristóbal de Las Casas, Chiapas, México; rrodiles@ecosur.mx

Received 14-III-2008. Corrected 11-VII-2008. Accepted 14-VIII-2008.

\begin{abstract}
Ichthyofauna of karstic wetlands under anthropic impact: the "petenes" of Campeche, Mexico. "Petenes" are small springs and associated streams that drain into wetlands near the coast in karstic areas. We studied composition, distribution, and abundance of the ichthyofauna in Los Petenes region (northwest Campeche). Two petenes displaying different degrees and types of anthropic impact were selected, Hampolol and El Remate. Hampolol has a smaller area but a longer derived stream; it is located within a protected area, but has been invaded by tilapia. El Remate is a popular spa, with no tilapia; it has a larger area but a shorter derived stream. At each "petén", several sites in the main spring, the associated stream, and secondary (temporary) springs were sampled in the rainy and dry seasons. Fishing gear was variable (throw net, gill net, small and large seine nets), but effort was uniform. We recorded temperature, dissolved oxygen, salinity, and depth at each site and season; also, we noted the different types and intensities of anthropic impact (channelization, presence of exotic species, recreational use, etc.) at each petén. We compared the petenes in terms of their environmental quality and fish fauna (composition, distribution, abundance, biomass); we also tested for effects of season and site within each petén. The study found 27 species of fishes, included in 18 genera and eight families, 24 species in Hampolol and 20 in El Remate. The geographical range of 'Cichlasoma'salvini, Rivulus tenuis, Phallichthys fairweatheri, Xiphophorus hellerii, and X. maculatus is extended. The dominant species in both seasons was Astyanax (probable hybrids A. aeneus $\times$ altior at Hampolol, pure A. altior at El Remate), which contributed most of the abundance and biomass, together with Vieja synspila and Poecilia velifera. A significantly greater overall diversity (H'n=3.31) was recorded in Hampolol compared to El Remate (H'n=2.10). Cluster analysis of sites by species presence allowed distinction of two groupings within each petén: permanent waters (i.e., main spring, stream) vs. temporary sites (secondary springs, which dry out in winter). Environmental parameters (except salinity) presented significant differences by site and season; a canonical correspondence analysis indicated that the distribution of fish assemblages was influenced by the environmental parameters in both seasons. In terms of composition, historical factors play a role in the differences between the fish assemblages of the two petenes, especially concerning the presence of Astyanax hybrids and Xiphophorus spp. in the southern petén (Hampolol) and Poecilia velifera in the northern petén (El Remate). Rev. Biol. Trop. 57 (1-2): 141-157. Epub 2009 June 30.
\end{abstract}

Key words: species composition, anthropic impact, fish assemblages, petén, Campeche, Yucatan Peninsula. 
Los petenes (vocablo procedente del maya "país llano") son islas de vegetación arbórea tropical, de selva mediana perennifolia y subperennifolia y de manglar, inmersos en amplias zonas inundables de tipo pantanoso o de vegetación estructuralmente más baja. Se distribuyen en todo el borde de la península de Yucatán, pero son abundantes en la porción centro-oriental de Quintana Roo y en el noroeste del estado de Campeche, donde se encuentra el área de este estudio. Debido a la importancia ecológica de estos humedales únicos en el país, Los Petenes en Campeche fueron declarados Reserva de la Biósfera en 1999, con una superficie de casi 282,858 ha (DOF 1999).

Los factores abióticos, como el nivel del agua y la concentración de oxígeno disuelto, pueden ser incluso más importantes que los factores bióticos en el control del tamaño de las poblaciones de peces tropicales. De la misma manera, los patrones de estacionalidad del medio son un factor clave que afecta las interacciones de la vida de la comunidad. Otro factor importante es la complejidad del hábitat, que regula la diversidad de la comunidad de peces (Lowe-McConnell 1987).

En los humedales cársticos costeros, como en la región de Los Petenes en Campeche, se conoce poco la influencia de los factores anteriores sobre las comunidades de peces. Se han realizado estudios en ambientes similares en el sur de Quintana Roo (Schmitter-Soto y Gamboa-Pérez 1996), en la Reserva de la Biósfera de Sian Ka'an (Maya Martínez 1998), en dolinas inundadas (cenotes) y humedales costeros del norte de Yucatán y Quintana Roo (Schmitter-Soto 1998b, 1999) y los Everglades en la Florida (Kushlan 1976, 1980, Loftus y Kushlan 1987, Kobza et al. 2004).

Los estudios realizados se han enfocado con más frecuencia hacia la vegetación y algunos elementos de la fauna, como las aves (Mas y Correa 2000), mientras que el componente acuático no se ha estudiado a detalle, a pesar de su importancia como indicador de integridad biótica y salud ambiental (Lyons et al. 1995). Villalobos-Zapata (2004) mencionó 68 especies de peces en la reserva de Los Petenes, pero de ellos sólo siete de agua dulce, previamente enlistados por Carrillo-Navarro (1995, citado ibíd.) en el petén Hampolol. A pesar de ser un área protegida, Los Petenes ha sufrido diversas perturbaciones antropogénicas.

Por lo anterior, el presente trabajo pretende contribuir al conocimiento taxonómico (composición) y ecológico de los peces continentales de la reserva, mediante el estudio de las asociaciones de especies de peces que habitan en aguas permanentes o temporales en dos petenes de la región, denominados Hampolol y El Remate, sujetos a diversos tipos de impacto antropogénico. Asimismo, se relaciona la estacionalidad (época de secas y lluvias) y la influencia de las variables ambientales (temperatura, oxígeno disuelto, salinidad y profundidad) sobre la distribución y abundancia de los peces. Por último, se discute la importancia de los factores históricos en contraste con los ecológicos como explicación de las diferencias ictiofaunísticas entre estos dos sistemas.

La hipótesis general subyacente al estudio es que la estructura y composición de las asociaciones de peces en estos sistemas cambiarán en función de los factores hidrológicos, así como también histórico-geográficos, y a los distintos niveles de impacto antropogénico. Se esperaba que el petén de mayor área contuviera una diversidad mayor, y que a un impacto humano más intenso correspondiera una menor diversidad; asimismo, dado que la región se ubica aproximadamente en la misma latitud que Tulum en la costa del Caribe, donde se ha ubicado una frontera ictiogeográfica (Schmitter-Soto 2002), se esperaba encontrar diferencias en composición faunística entre ambos petenes, no sólo atribuibles a factores ecológicos.

\section{MATERIALES Y MÉTODOS}

Área de estudio: Los dos sistemas acuáticos en estudio forman parte de la región de Los Petenes, actualmente Reserva de la Biósfera, caracterizada por la presencia de "islas" de vegetación arbórea inmersas en una matriz de vegetación baja inundable. Estas "islas" 
(petenes), conformadas por vegetación de selva o manglar estructuralmente más alta y densa que el entorno, sólo se encuentran en la península de Yucatán, la Florida y Cuba. Su particular distribución se asocia con resurgencias de agua dulce sobre planicies cárstico-palustres (calizas del Cuaternario), las cuales propician el desarrollo de una red hidrológica particular, alrededor de la cual crece la vegetación con mayor vigor y densidad.

La estructura geológica superficial y el subsuelo demuestran que la plataforma que constituye la actual península de Yucatán inició su emersión lenta y gradual sobre el nivel del mar durante el Eoceno (Emery 1977). Después, en el Oligoceno y Mioceno inferior, se depositaron carbonatos, sobre todo en la porción norte, y fue hasta el Mioceno superior que reinició un levantamiento más importante en la porción meridional de la península, menos pronunciado en la porción norte y en el área de Los Petenes (López-Ramos 1981, Duch 1988).

Debido a su cercanía con el mar y a la naturaleza hidrogeológica cárstica del terreno, los petenes son biotopos con diversos hábitats: ojos de agua que evolucionan hacia dolinas inundadas (cenotes), ojos de agua temporales o permanentes, canales angostos de intercomunicación entre los ojos de agua, y canales de marea. Además, el área y volumen de estos sistemas tienden a variar a lo largo del año y entre años, debido principalmente al régimen de mareas, las lluvias de verano, las lluvias extraordinarias de tormenta por temporales, huracanes y nortes, la obstrucción natural o inducida de canales y el azolve gradual por sedimentos calcáreos en algunos ojos de agua.

La inundabilidad de los sistemas hidrológicos está condicionada por las épocas climáticas. En general, la época de secas en esta región va de noviembre a abril, la de lluvias de mayo a octubre, y la de nortes de noviembre a febrero. En la zona norte, la temperatura media anual es de $27.8{ }^{\circ} \mathrm{C}$ y la precipitación es de 700-800 mm anuales, mientras que en el sur la temperatura decrece a $26.4{ }^{\circ} \mathrm{C}$ y la precipitación llega a 800-1100 mm (YáñezArancibia et al. 1996).
El primero de los petenes, Hampolol, se ubica en el predio del Centro para la Conservación e Investigación de la Vida Silvestre, bajo resguardo de la Universidad Autónoma de Campeche en el municipio y estado del mismo nombre en la República Mexicana (19'56'36" N, 90²2’31" W) (Fig. 1). El área de su manantial principal se estima en $177 \mathrm{~m}^{2}$, con una longitud de $129 \mathrm{~m}$ en su principal canal efluente (desde el borde del manantial hasta la desembocadura en un canal mayor). El segundo petén, El Remate, se encuentra unos $60 \mathrm{~km}$ al norte del anterior, en el municipio de Calkiní, mismo estado de la República (20³2’04” N, 90²2'13” W) (Fig. 1). El área de su manantial principal es mayor $\left(491 \mathrm{~m}^{2}\right)$, aunque el canal efluente es más corto (92 m). La distancia al mar en línea recta es similar en ambos petenes: $8.3 \mathrm{~km}$ en Hampolol, $11.7 \mathrm{~km}$ en el caso de El Remate.

Aunque los dos petenes en estudio presentan perturbación antropogénica, se pueden diferenciar sus tipos e intensidades. En Hampolol el acceso es restringido, por lo cual no hay una presión significativa de uso recreativo; sin embargo, se detectó la presencia de especies exóticas en el sistema. Por otra parte, a pesar de que El Remate presenta una presión antrópica cada vez mayor (últimamente se ha convertido en el balneario ejidal "natural" más concurrido del norte de Campeche y sur de Yucatán), en este sistema no se capturó tilapia.

Trabajo de campo: La comunidad de peces se muestreó por meses en cada uno de los petenes durante las épocas de secas (noviembre-abril) y lluvias (mayo-octubre) del año 2003. En cada petén se distinguieron a priori tres hábitats: ojos de agua permanentes, ojos de agua temporales (los cuales desaparecieron en la época de secas) y canal de intercomunicación. En cada hábitat se midieron durante las horas de la mañana (9-12 am) temperatura, salinidad y concentración de oxígeno disuelto con ayuda de un multianalizador YSI 85, así como la profundidad, con una vara metrada.

Para la captura de los especímenes se usaron diversos artes de pesca, según las 


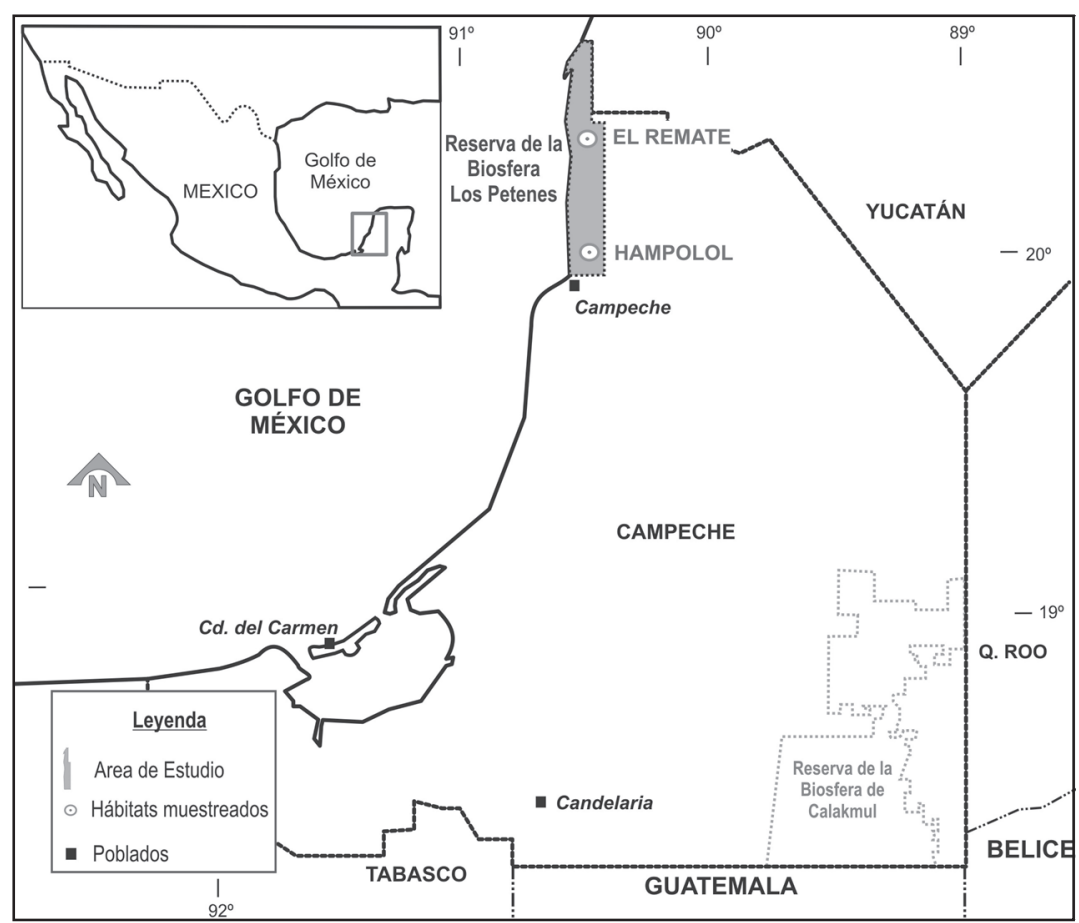

Fig. 1. Área de estudio y hábitats muestreados. / Fig. 1. Study area and sampled habitats.

condiciones de cada hábitat: chinchorro playero $(20 \mathrm{~m}$ de largo $\times 1.5 \mathrm{~m}$ de alto, con una luz de malla de $1 \mathrm{~cm}$ ), chinchorro pequeño (3 $\mathrm{m}$ de largo $\times 1 \mathrm{~m}$ de alto, luz de malla de 0.5 $\mathrm{cm}$ ), atarraya (3 $\mathrm{m}$ de diámetro, luz de malla de $2.5 \mathrm{~cm}$ ) y red agallera (utilizada de manera estacionaria, por dos horas; $7 \mathrm{~m}$ de largo $\times 1.5$ $\mathrm{m}$ de alto, luz de malla de $3 \mathrm{~cm}$ ). Sólo la atarraya y el chinchorro pequeño fueron aplicables en todos los hábitats, de modo que su uso fue estandarizado a tres lances cada arte. El área estimada cubierta por la atarraya fue de $21 \mathrm{~m}^{2}$; el área de barrido del chinchorro pequeño se calculó en $11 \mathrm{~m}^{2}$.

Los individuos capturados se colocaron en bolsas de plástico etiquetadas con los datos de recolección y fueron fijados en formol al 10\%. En el laboratorio se preservaron en alcohol al 70\% y se identificaron con la ayuda de claves (Greenfield y Thomerson 1997, Schmitter-Soto 1998a). Una vez determinadas las especies, se contó el número de ejemplares y se registró el peso total y la longitud estándar de manera individual.
Los ejemplares fueron depositados en la colección de peces de ECOSUR, unidades San Cristóbal y Chetumal, y en el CINVESTAV, Unidad Mérida; los números de catálogo están a disposición de los interesados.

Análisis de datos: Los datos de cada arte de pesca (atarraya y chinchorro pequeño) se analizaron de manera separada. La densidad y biomasa se determinaron como el número de individuos y peso registrados por metro cuadrado.

La estructura de la comunidad se analizó a nivel espacial y temporal considerando los principales descriptores: riqueza de especies $(S)$, diversidad $\left(H^{\prime}\right)$ de Shannon-Wiener, equidad $(E)$ y dominancia (Índice de Valor de Importancia, IVI, el cual incorpora información sobre la densidad, la biomasa relativa y frecuencia de cada especie) (de la Cruz 1994). Previa verificación de las suposiciones de normalidad (prueba de Kolmogorov-Smirnov) y homoscedasticidad de los datos (prueba de 
Bartlett) (Underwood 1997), se llevó a cabo un análisis de varianza (ANOVA) de tres vías (hábitat, petenes, épocas climáticas). De igual manera se procedió con las variables ambientales (temperatura, oxígeno disuelto, salinidad y profundidad).

Se realizó un análisis de conglomerados para determinar los conjuntos de especies y sitios. Para ello se eligió el índice de Bray-Curtis, por su precisión al reflejar la similitud en la composición de especies y su relación con la abundancia, dando mayor peso a las especies más abundantes (Bloom 1981), así como el agrupamiento medio no ponderado (UPGMA) (Cuadro 1).

La relación entre la densidad de las especies y las variables ambientales se estimó también mediante un Análisis de Correspondencia Canónica (ACC).

Para la estimación de los descriptores de la comunidad, así como para el análisis de agrupación, se utilizó el programa estadístico denominado ANACOM (de la Cruz 1994); el ACC, en el programa MVSP; los ANOVA, con el programa STATISTICA para Windows, versión 5.5. En todas las pruebas estadísticas, $p \leq 0.05$.

\section{RESULTADOS}

Variables ambientales: La temperatura y oxígeno disuelto mostraron diferencias significativas $(\mathrm{F}=2.44, p<0.05 ; \mathrm{F}=10.14, p$ $<<0.01$, respectivamente) entre los hábitats al interior de cada petén. Los canales fueron más cálidos y mejor oxigenados que los ojos de agua (27.6-31.8 ${ }^{\circ} \mathrm{C}$ vs. $26.0-27.9^{\circ} \mathrm{C} ; 2.8-8.6 \mathrm{mg} / \mathrm{l}$ vs. $1.6-4.8 \mathrm{mg} / \mathrm{l})$. La profundidad difirió entre los hábitats $(\mathrm{F}=64.85, p<<0.01)$, con $0.0-0.3 \mathrm{~m}$ en los ojos de agua temporales, 0.7-0.9 $\mathrm{m}$ en los permanentes y $0.5-1.3$ en los canales.

Entre ambos petenes sólo resultó significativa la diferencia de salinidad ( $\mathrm{F}=31.24, p<<$ $0.01)$ : El Remate llega a ser más salobre que el petén Hampolol (hasta 3.1\%o, vs. 0.6-2.1\%o).

Composición específica: En ambos petenes, la comunidad íctica estuvo representada por peces jóvenes y adultos. Se capturó un total de 842 individuos en el petén Hampolol y 1134 en el petén El Remate. En Hampolol se identificaron 24 especies y en El Remate 20; la riqueza combinada fue de 27 especies (Cuadro 2).

El porcentaje de especies de origen marino fue de un $8 \%$ en Hampolol ( 2 de 24), mientras que en El Remate fue del 20\% (4 de 20). En Hampolol se encontraron dos especies de tilapia, mientras que en El Remate no se detectó ninguna especie exótica.

La identificación de las sardinitas dulceacuícolas del género Astyanax fue problemática en el petén Hampolol; 63\% de los ejemplares se

CUADRO 1

Variables ambientales del petén Hampolol (Ha) y del petén El Remate (Re)

TABLE 1

Environmental variables of petén Hampolol (Ha) and petén El Remate (Re)

\begin{tabular}{|c|c|c|c|c|c|c|c|c|}
\hline \multirow{3}{*}{ Sitio de muestreo } & \multirow{2}{*}{\multicolumn{2}{|c|}{ Temperatura $\left({ }^{\circ} \mathrm{C}\right)$}} & \multirow{2}{*}{\multicolumn{2}{|c|}{ Salinidad }} & \multirow{2}{*}{\multicolumn{2}{|c|}{$\begin{array}{l}\text { Oxígeno disuelto } \\
\text { (mg/l) }\end{array}$}} & \multirow{2}{*}{\multicolumn{2}{|c|}{$\begin{array}{l}\text { Profundidad } \\
\text { (m) }\end{array}$}} \\
\hline & & & & & & & & \\
\hline & Secas & Lluvias & Secas & Lluvias & Secas & Lluvias & Secas & Lluvias \\
\hline Ojo de agua permanente $(\mathrm{Ha})$ & 27.4 & 28 & 1.3 & 1.2 & $3.2-3.7$ & $3.8 .-4.6$ & $0.7-0.8$ & $0.7-0.8$ \\
\hline Ojo de agua temporal ( $\mathrm{Ha})$ & 26.7 & 27.7 & 1.28 & 1 & 3 & 4.61 & 0.1 & 0.3 \\
\hline Canal (Ha) & $28-29$ & $28-30$ & 1.3 & $1.1-1.2$ & $3.5-7.8$ & $4.3-8$ & $0.3-1.0$ & $0.4-1.0$ \\
\hline Promedio Hampolol & 27.6 & 28.2 & 1.2 & 1.2 & 4.2 & 5.1 & 0.6 & 0.7 \\
\hline Ojo de agua permanente (Re) & 27 & 27 & 2 & 2 & $4-4.8$ & $3-3.4$ & $0.9-0.7$ & $0.8-1.1$ \\
\hline Ojo de agua temporal (Re) & 27.8 & 27.4 & 1.8 & 1.3 & 4.1 & 4.6 & 0.2 & 0.2 \\
\hline Canal (Re) & $26-28$ & $27-30$ & $2-2.1$ & $2-2.5$ & $3.1-4.2$ & $3-5.8$ & $0.5-0.7$ & $0.8-0.6$ \\
\hline Promedio El Remate & 27.3 & 28 & 2 & 2 & 4.1 & 4 & 0.6 & 0.7 \\
\hline
\end{tabular}


CUADRO 2

Lista de los peces de los petenes Hampolol y El Remate, Campeche, México. Se marcan con asterisco las especies registradas previamente en Hampolol por Carrillo-Navarro (1995, citado por Villalobos-Zapata 2004)

TABLE 2

Checklist of fishes from the petenes Hampolol and El Remate, Campeche, Mexico. The asterisks mark species previously recorded at Hampolol by Carrillo-Navarro (1995, cited by Villalobos-Zapata 2004)

Clase Actinopterygii

Orden Elopiformes

Familia Megalopidae

Megalops atlanticus Valenciennes, 1847

Orden Characiformes

Familia Characidae

Astyanax aeneus (Günther, 1860)*

Astyanax altior Hubbs, 1936

Astyanax aeneus $\times$ altior (posibles híbridos)

Orden Siluriformes

Familia Heptapteridae

Rhamdia guatemalensis (Günther, 1864)*

Orden Beloniformes

Familia Belonidae

Strongylura timucu (Walbaum, 1792)

Orden Cyprinodontiformes

Familia Aplocheilidae

Rivulus tenuis Meek, 1904

Familia Poeciliidae

Belonesox belizanus Kner, 1860*

Gambusia yucatana Regan, 1914*

Gambusia sexradiata Hubbs, 1936

Heterandria bimaculata (Heckel, 1848)

Phallichthys fairweatheri Rosen y Bailey, 1959

Poecilia mexicana Steindachner, 1863

Poecilia velifera (Regan, 1914)

Xiphophorus hellerii Heckel, 1848

Xiphophorus maculatus (Günther, 1866)

Orden Perciformes

Familia Gerreidae

Eucinostomus argenteus Baird y Girard, 1855

Gerres cinereus (Walbaum, 1792)

Familia Cichlidae

Astatheros robertsoni (Regan, 1905)

'Cichlasoma'salvini (Günther, 1862)

'Cichlasoma' urophthalmus (Günther, 1862)*

Oreochromis aureus (Steindachner, 1864)

Oreochromis niloticus (Linnaeus, 1758)

Petenia splendida Günther, 1862*

Rocio octofasciata (Regan, 1903)

Thorichthys meeki Brind, 1918*

Vieja synspila (Hubbs, 1935)

consideraron como posibles híbridos A. aeneus $\times$ A. altior, con un $35 \%$ asignable a $A$. altior y sólo un $2 \%$ a $A$. aeneus. En el petén más septentrional, El Remate, todos los Astyanax correspondieron a A. altior.
Hampolol El Remate

X

X

X

X $\quad X$

$\mathrm{X}$

X

X

X

X $\quad$ X

X $\quad X$

$\mathrm{X}$

X X

X X

X $\quad X$

X $\quad X$

$\mathrm{X}$

X $\quad X$

$\begin{array}{ll}\mathrm{X} & \mathrm{X}\end{array}$

X $\quad X$

$\mathrm{X} \quad \mathrm{X}$

$\mathrm{X} \quad \mathrm{X}$

$\mathrm{X}$

$\mathrm{X}$

$\mathrm{X}$

$\mathrm{X} \quad \mathrm{X}$

$\mathrm{X} \quad \mathrm{X}$

$\mathrm{X} \quad \mathrm{X}$ 
CUADRO 3

Variables ecológicas de las comunidades ícticas del petén Hampolol (Ha) y El Remate (Re).

$S=$ riqueza, $H^{\prime}=$ diversidad (bits $\cdot$ ind $^{-1}$ ), $E=$ equidad.

TABLE 3

Ecological variables of the fish communities at petén Hampolol (Ha) and El Remate (Re). $S=$ richness, $H^{\prime}=$ diversity $\left(\right.$ bits $\cdot$ ind $\left.^{-1}\right), E=$ equitability

\begin{tabular}{|c|c|c|c|c|c|c|c|c|c|}
\hline \multirow{3}{*}{ Hábitat } & \multicolumn{3}{|c|}{$S$} & \multicolumn{3}{|c|}{$H^{\prime}$} & \multicolumn{3}{|c|}{$E$} \\
\hline & \multirow{2}{*}{ Espacial } & \multicolumn{2}{|c|}{ Temporal } & \multirow{2}{*}{ Espacial } & \multicolumn{2}{|c|}{ Epocas } & \multirow{2}{*}{ Espacial } & \multicolumn{2}{|c|}{ Epocas } \\
\hline & & Secas & Lluvias & & Secas & Lluvias & & Secas & Lluvias \\
\hline Ojo de agua permanente $(\mathrm{Ha})$ & $13-16$ & 11 & $8-11$ & $2.0-2.7$ & $2.1-1.7$ & $2.3-3.0$ & $0.5-0.7$ & $0.5-0.6$ & $0.8-0.9$ \\
\hline Ojo de agua temporal (Ha) & 10 & 6 & 8 & 2.4 & 1.7 & 2.6 & 0.7 & 0.7 & 0.9 \\
\hline Canal (Ha) & 8 & 5 & 8 & 2.0 & 1.2 & 2.2 & 0.7 & 0.5 & 0.7 \\
\hline Total Hampolol & 24 & 23 & 19 & 3.3 & 3.1 & 3.3 & 0.7 & 0.7 & 0.8 \\
\hline Ojo de agua permanente (Re) & $7-9$ & $4-9$ & $5-7$ & $1.2-1.6$ & $0.8-2.0$ & $1.1-1.3$ & $0.4-0.5$ & $0.4-0.6$ & 0.5 \\
\hline Ojo de agua temporal (Re) & 9 & 5 & 9 & 2.4 & 2.0 & 2.3 & 0.8 & 0.8 & 0.7 \\
\hline Canal (Re) & $8-10$ & $8-10$ & 5 & $1.4-1.8$ & $1.2-2.0$ & $1.0-1.1$ & $0.5-0.6$ & $0.4-0.6$ & $0.4-0.5$ \\
\hline Total El Remate & 20 & 18 & 18 & 2.10 & 1.84 & 2.16 & 0.48 & 0.44 & 0.51 \\
\hline
\end{tabular}

3 ), incluso sin tomar en cuenta la presencia de dos especies exóticas. No hubo diferencias por época ni por hábitat.

De acuerdo con el $I V I$, las especies dominantes fueron las del género Astyanax: $A$. aeneus $\times$ altior en Hampolol (15.9 \%) y $A$. altior en El Remate (38.6 \%). La segunda especie en dominancia en Hampolol fue Thorichthys meeki (11.5\%); en El Remate, Poecilia velifera (17.1\%). En ambos petenes, el tercer lugar correspondió a Poecilia mexicana (9.0\% y $8.9 \%$, respectivamente) (Cuadro 4$)$.

Variación espacio-temporal de la densidad y biomasa: En el petén Hampolol, la familia Poeciliidae fue la más abundante en la época de secas, con Gambusia sexradiata como la especie de mayor densidad. Le siguieron en importancia las familias Cichlidae y Characidae, en especial T. meeki y los híbridos de Astyanax, respectivamente. Por otra parte, la familia Characidae fue la más abundante en la época de lluvias, seguida por las familias Poeciliidae y Cichlidae (Cuadro 5A).

En cuanto a la biomasa, los cíclidos y carácidos registraron los valores máximos en las dos épocas (Cuadro 5B). En secas, A. aeneus $\times$ altior, T. meeki y Vieja synspila presentaron los porcentajes de biomasa más altos, mientras que en lluvias $V$. synspila y Oreochromis niloticus estuvieron mejor representadas.

En El Remate, en secas y lluvias A. altior y especies de Poeciliidae tuvieron las mayores densidades y biomasas(Cuadro 6), seguidos por $T$. meeki. $P$. velifera fue más abundante en la época de secas, mientras que $P$. mexicana y $G$. sexradiata lo fueron durante las lluvias. $A$. altior presentó el $43 \%$ de la biomasa relativa en secas y 49\% en lluvias (Cuadro 6B).

No hubo diferencias significativas entre la densidad de peces en ambos petenes: 1.1-2.2 ind $\times \mathrm{m}^{2}$ en Hampolol, 1.6-2.4 ind $\times \mathrm{m}^{2}$ en El Remate. Tampoco las hubo entre las épocas climáticas, ni en la interacción petén-época.

Tanto en Hampolol como El Remate, la densidad no presentó diferencias significativas entre los hábitats, pero la biomasa sí lo hizo. En Hampolol, el hábitat con menor biomasa relativa (3\%) fue el ojo de agua temporal. Los ojos de agua permanentes tuvieron las mayores biomasas relativas (26-53\%). En El Remate los hábitats que sobresalieron fueron el canal $(35 \%)$ y el ojo de agua permanente (25\%); los valores más bajos se registraron en el ojo 
CUADRO 4

Especies dominantes de la comunidad de peces asociadas a los petenes Hampolol y El Remate a partir del índice de valor de importancia (IVI)

TABLE 4

Dominant species of the fish community associated to the petenes Hampolol and El Remate based on the Index of Importance Value (IVI)

\begin{tabular}{|c|c|c|c|}
\hline Especie & Petén Hampolol IVI (\%) & Especie & $\begin{array}{c}\text { Petén El Remate } \\
\text { IVI }(\%)\end{array}$ \\
\hline Astyanax aeneus $\cdot$ altior & 15.9 & Astyanax altior & 38.6 \\
\hline Thorichthys meeki & 11.5 & Poecilia velifera & 17.1 \\
\hline Poecilia mexicana & 9 & Poecilia mexicana & 8.9 \\
\hline Vieja synspila & 8.9 & Thorichthys meeki & 6.6 \\
\hline Astyanax altior & 8.8 & 'Cichlasoma' urophthalmus & 3.5 \\
\hline Oreochromis niloticus & 8.5 & 'Cichlasoma'salvini & 3.0 \\
\hline Gambusia sexradiata & 7.1 & Gambusia sexradiata & 2.9 \\
\hline Phallichthys fairweatheri & 3.9 & Belonesox belizanus & 2.8 \\
\hline Astyanax aeneus & 3.2 & Strongylura timuсu & 2.6 \\
\hline Heterandria bimaculata & 2.5 & Rocio octofasciata & 1.9 \\
\hline Oreochromis aureus & 2.4 & Astatheros robertsoni & 1.8 \\
\hline Xiphophorus maculatus & 2.2 & Megalops atlanticus & 1.7 \\
\hline Petenia splendida & 2 & Rhamdia guatemalensis & 1.7 \\
\hline 'Cichlasoma' urophthalmus & 2 & Eucinostomus argenteus & 1.2 \\
\hline 'Cichlasoma' salvini & 1.8 & Phallichthys fairweatheri & 1.2 \\
\hline Belonesox belizanus & 1.8 & Heterandria bimaculata & 1.1 \\
\hline Xiphophorus hellerii & 1.7 & Rivulus tenuis & 0.9 \\
\hline Rocio octofasciata & 1.6 & Gerres cinereus & 0.9 \\
\hline Rhamdia guatemalensis & 1.5 & Xiphophorus maculatus & 0.8 \\
\hline Rivulus tenuis & 1.2 & Vieja synspila & 0.8 \\
\hline Gambusia yucatana & 1 & & \\
\hline Eucinostomus argenteus & 1 & & \\
\hline Astatheros robertsoni & 0.8 & & \\
\hline Total & 100 & & 100 \\
\hline
\end{tabular}

de agua temporal $(9 \%)$, igual que en el otro petén.

Relación especie-ambiente: Tanto en Hampolol como en El Remate, el análisis de conglomerados definió dos grupos: uno formado por los ojos de agua permanentes y el canal, y el otro sólo por el ojo de agua temporal. El primer grupo se caracteriza por la frecuencia y densidad de Astyanax, T. meeki, Poecilia y $V$. synspila; el segundo grupo, por especies de talla pequeña (hasta $3 \mathrm{~cm}$ ), asociadas a fondos lodosos de poca profundidad y con vegetación en descomposición, por ejemplo Rivulus tenuis, las dos especies de Gambusia, Heterandria bimaculata, Phallichthys fairweatheri y Xiphophorus maculatus.

El ACC confirmó y precisó el patrón definido por el análisis de conglomerados: se distinguieron de manera clara los ojos de agua temporales de ambos petenes, por su baja profundidad y concentración de oxígeno disuelto y por su composición de especies (ver abajo), mientras que el resto de los sitios de muestreo (ojos de agua permanentes, canales) se agruparon según la localidad (petén): los hábitats 
CUADRO 5

Petén Hampolol: Densidad (A) y biomasa relativa (B) de peces por familia en época seca y de lluvias

TABLE 5

Petén Hampolol: Density (A) and relative biomass (B) of fishes by family in the dry and rainy seasons

$\mathbf{A}$

\begin{tabular}{lcclcc}
\multicolumn{1}{c}{ Familia } & $\begin{array}{c}\text { Secas } \\
\text { Número total de } \\
\text { especies }\end{array}$ & Densidad & Familia & $\begin{array}{c}\text { Lluvias } \\
\text { Número total de } \\
\text { especies }\end{array}$ & Densidad \\
\hline Poeciliidae & 8 & 40.3 & Characidae & 3 & 39.1 \\
Characidae & 3 & 36.5 & Poeciliidae & 6 & 38.6 \\
Cichlidae & 7 & 21.4 & Cichlidae & 7 & 1 \\
Aplocheilidae & 1 & 1.6 & Gerreidae & 1 & 1.1 \\
Heptapteridae & 1 & 0.2 & Aplocheilidae & 18 & 0.7 \\
Total & 20 & 100 & & & 100
\end{tabular}

B

\begin{tabular}{|c|c|c|c|c|c|}
\hline \multicolumn{3}{|c|}{ Secas } & \multicolumn{3}{|c|}{ Lluvias } \\
\hline Familia & $\begin{array}{c}\text { Número total de } \\
\text { especies }\end{array}$ & $\begin{array}{l}\text { Porcentaje de } \\
\text { biomasa }\end{array}$ & Familia & $\begin{array}{c}\text { Número total de } \\
\text { especies }\end{array}$ & $\begin{array}{c}\text { Porcentaje de } \\
\text { biomasa }\end{array}$ \\
\hline Cichlidae & 7 & 44.7 & Cichlidae & 7 & 66.4 \\
\hline Characidae & 3 & 35.0 & Characidae & 3 & 20.3 \\
\hline Poeciliidae & 8 & 14.6 & Poeciliidae & 6 & 12.4 \\
\hline Heptapteridae & 1 & 5.3 & Gerreidae & 1 & 0.8 \\
\hline Aplocheilidae & 1 & 0.4 & Aplocheilidae & 1 & 0.0 \\
\hline Total & 20 & 100 & & 18 & 100 \\
\hline
\end{tabular}

permanentes del petén Hampolol se correlacionaron con la temperatura, profundidad y oxígeno disuelto, mientras que los hábitats permanentes de El Remate se agruparon por la salinidad y en segundo lugar por la profundidad (Fig. 2). En toda época, en el eje uno la variable mejor correlacionada fue la salinidad, y en el eje dos la profundidad; entre los dos primeros componentes se explicó un 53 a $56 \%$ de la variación (Cuadro 7).

Ahora bien, en secas fue evidente una alta correlación entre el oxígeno disuelto y la profundidad (Fig. 2A), mientras que en lluvias no ocurrió así (Fig. 2B). Por otra parte, la relación entre la profundidad y la temperatura aumentó durante la época lluviosa.

Los patrones de asociación entre las especies y su relación con las variables ambientales observados en secas fueron los siguientes:
Las especies $A$. aeneus $\times$ altior, T. meeki, Xiphophorus hellerii, V. synspila y O. niloticus mostraron una distribución en gradientes de menor a mayor con respecto a la temperatura, oxígeno disuelto y profundidad.

Astatheros robertsoni, Rocio octofasciata, Rhamdia guatemalensis, 'Cichlasoma' salvini, Belonesox belizanus y P. mexicana se relacionaron con valores superiores al promedio en cuanto a profundidad, oxígeno disuelto y temperatura.

'C.' urophthalmus, A. altior, P. velifera y Strongylura timucu estuvieron relacionadas principalmente con la salinidad.

Gambusia yucatana, $G$. sexradiata y $R$. tenuis se asociaron con baja profundidad y baja concentración de oxígeno disuelto, características propias de los hábitats temporales.

En lluvias se interpretaron cinco grupos de especies, según el ACC. Las especies T. meeki, 
CUADRO 6

Petén El Remate: Densidad (A) y biomasa relativa (B) de peces por familia en época de secas y lluvias

TABLE 6

Petén El Remate: Density $(\boldsymbol{A})$ and relative biomass $(\boldsymbol{B})$ of fishes by family in the dry and rainy seasons

A

\begin{tabular}{|c|c|c|c|c|c|}
\hline \multicolumn{3}{|c|}{ Secas } & \multicolumn{3}{|c|}{ Lluvias } \\
\hline Familia & $\begin{array}{c}\text { Número total de } \\
\text { especies }\end{array}$ & Densidad & Familia & $\begin{array}{c}\text { Número total de } \\
\text { especies }\end{array}$ & Densidad \\
\hline Characidae & 1 & 64.7 & Characidae & 1 & 59.5 \\
\hline Poeciliidae & 5 & 24.9 & Poeciliidae & 7 & 33.2 \\
\hline Cichlidae & 6 & 8.8 & Cichlidae & 4 & 4.3 \\
\hline Heptapteridae & 1 & 0.4 & Gerreidae & 2 & 1.6 \\
\hline Aplocheilidae & 1 & 0.4 & Belonidae & 1 & 0.8 \\
\hline Gerreidae & 2 & 0.4 & Aplocheilidae & 1 & 0.4 \\
\hline Belonidae & 1 & 0.4 & Megalopidae & 1 & 0.3 \\
\hline Total & 17 & 100 & & 17 & 100 \\
\hline
\end{tabular}

\section{B}

\begin{tabular}{|c|c|c|c|c|c|}
\hline \multicolumn{3}{|c|}{ Secas } & \multicolumn{3}{|c|}{ Lluvias } \\
\hline Familia & $\begin{array}{c}\text { Número total de } \\
\text { especies }\end{array}$ & $\begin{array}{l}\text { Porcentaje de } \\
\text { biomasa }\end{array}$ & Familia & $\begin{array}{c}\text { Número total de } \\
\text { especies }\end{array}$ & $\begin{array}{c}\text { Porcentaje de } \\
\text { biomasa }\end{array}$ \\
\hline Characidae & 1 & 43.0 & Characidae & 1 & 49.0 \\
\hline Poeciliidae & 5 & 37.6 & Poeciliidae & 7 & 31.8 \\
\hline Cichlidae & 6 & 14.8 & Cichlidae & 4 & 9.7 \\
\hline Belonidae & 1 & 4.5 & Megalopidae & 1 & 5.6 \\
\hline Heptapteridae & 1 & 0.4 & Belonidae & 1 & 2.9 \\
\hline Gerreidae & 2 & 0.1 & Gerreidae & 2 & 1.3 \\
\hline Aplocheilidae & 1 & 0.0 & Aplocheilidae & 1 & 0.0 \\
\hline Total & 17 & 100 & & 17 & 100 \\
\hline
\end{tabular}

\section{CUADRO 7}

Análisis de Correspondencia Canónica de la comunidad de peces en los petenes Hampolol y El Remate durante las épocas de secas y lluvias

TABLE 7

Canonical Correspondence Analysis of the fish community in the petenes Hampolol and El Remate during the dry and rainy season

\begin{tabular}{|lccccc}
\multicolumn{1}{c}{ Resultados } & \multicolumn{2}{c}{ Secas } & \multicolumn{2}{c}{ Lluvias } \\
& eje 1 & eje 2 & eje 1 & eje 2 \\
\hline Eigenvalores & 0.78 & 0.44 & 0.68 & 0.42 \\
Porcentaje explicado de la variancia & 35.9 & 20.1 & 32.8 & 20.0 \\
Correlación entre las variables ambientales y las especies & 0.91 & 0.96 & 0.99 & 0.97 \\
Coeficiente canónico & -1.14 & -0.92 & -0.94 & -1.03
\end{tabular}




\section{A}

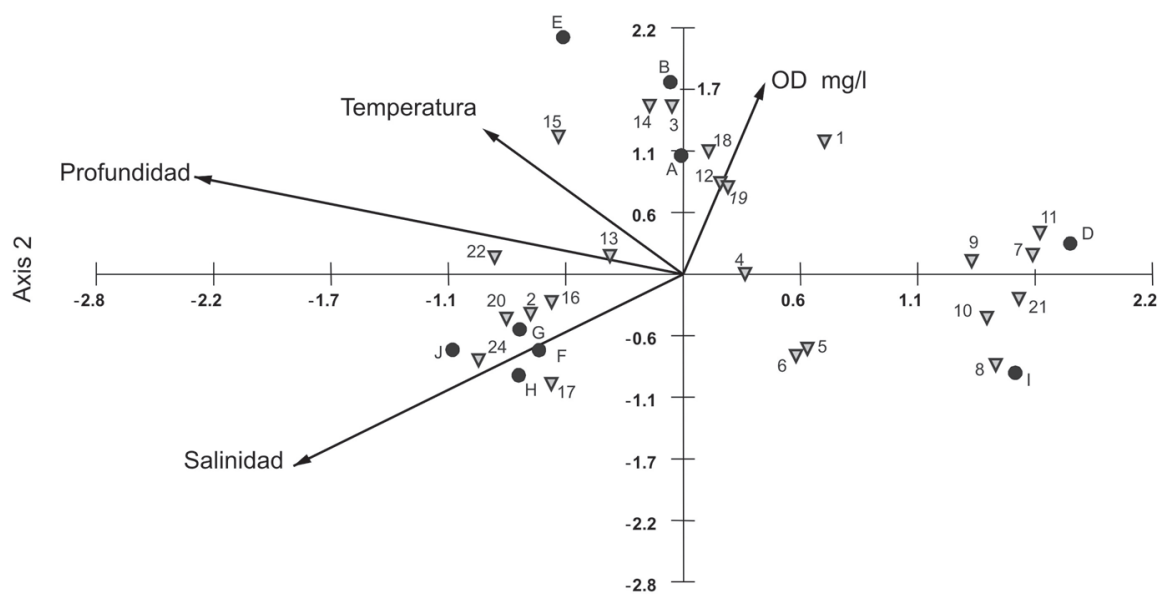

Axis 1

B

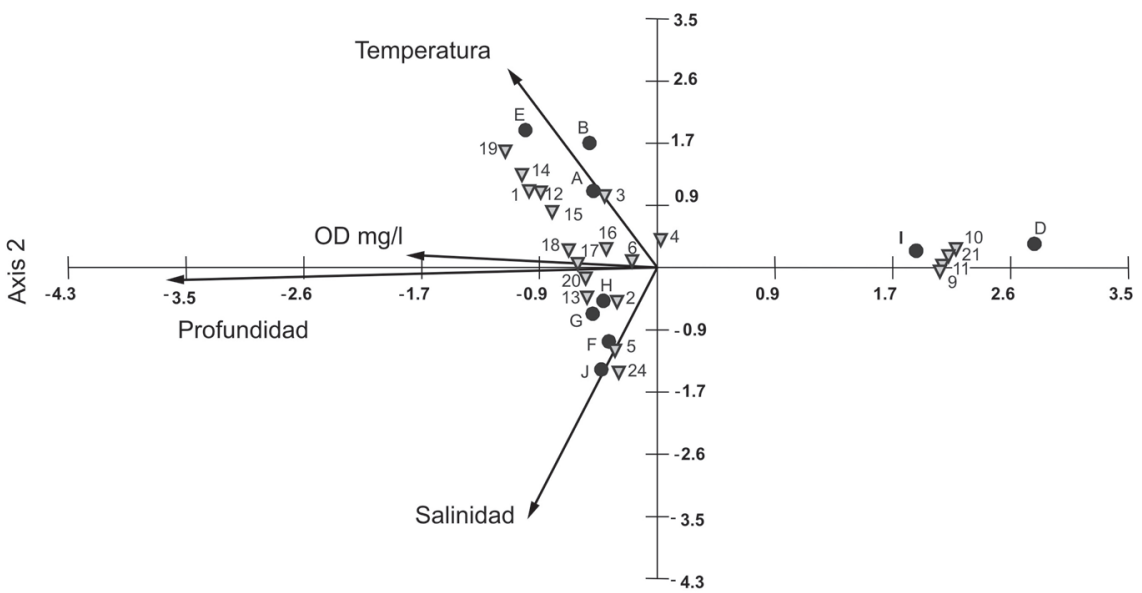

Axis 1

Fig. 2. Análisis de correspondencia canónica entre las asociaciones de peces y las variables ambientales en la época de secas (A) y lluvias (B) en los petenes de Hampolol y El Remate. Petenes: A, B, D, E- Hampolol; F, G, H, I, J- El Remate. Hábitats: A, B, F, G- ojos de agua permanentes; D, I, ojos de agua temporales; E, H, J, canales.

Especies: 1- A. aeneus, 2- A. altior, 3- A. aeneus $\times$ altior, 4- P. mexicana, 5- P. velifera, 6- B. belizanus, 7- H. bimaculata, 8-X. maculatus, 9- P. fairweatheri, 10- G. yucatana, 11- G. sexradiata, 12- X. helleri, 13- 'C. 'urophthalmus, 14- V. synspila, 15- T. meeki, 16- 'C.' salvini, 17- R. octofasciata, 18- A. robertsoni, 19- O. niloticus, 20- R. guatemalensis, 21- R. tenuis, 22- E. argenteus, 23- G. cinereus, 24- S. timucu.

Fig. 2. Canonic Correspondence Analysis between fish assemblages and environmental variables during the dry season (A) and the rainy season (B) in the petenes Hampolol and El Remate. Petenes: A, B, D, E- Hampolol; F, G, H, I, J- El Remate. Habitats: A, B, F, G- permanent springs; D, I, temporary springs; E, H, J, canals.

Species: 1- A. aeneus, 2- A. altior, 3- A. aeneus $\times$ altior, 4- P. mexicana, 5- P. velifera, 6- B. belizanus, 7- H. bimaculata, 8-X. maculatus, 9- P. fairweatheri, 10- G. yucatana, 11- G. sexradiata, 12-X. helleri, 13- 'C. 'urophthalmus, 14- V. synspila, 15- T. meeki, 16- 'C.' 'salvini, 17- R. octofasciata, 18- A. robertsoni, 19- O. niloticus, 20- $R$. guatemalensis, 21- R. tenuis, 22- E. argenteus, 23- G. cinereus, 24- S. timucu. 
V. synspila, A. aeneus $\times$ altior, Oreochromis aureus, $O$. niloticus y $A$. aeneus presentaron una correlación alta con el oxígeno disuelto y cercana a la media para la temperatura y la profundidad, a excepción de T. meeki, que mostró una alta correlación con la temperatura, y $A$. aeneus, que presentó una correlación por debajo de la media con la temperatura y la profundidad.

Las especies 'C.' urophthalmus y Eucinostomus argenteus estuvieron relacionadas principalmente con la profundidad.

Las especies ' $C$.' salvini, A. altior, Megalops atlanticus, $A$. robertsoni y $S$. timucu se asociaron con la salinidad y profundidad.

Las densidades de las especies $P$. mexicana, $P$. velifera y $B$. belizanus estuvieron determinadas por valores de profundidad y temperatura por debajo de la media.

Las especies $X$. hellerii, $H$. bimaculata, $P$. fairweatheri, $R$. tenuis, $G$. sexradiata y $X$. maculatus se asociaron a los valores de profundidad más bajos de los sistemas en estudio.

\section{DISCUSIÓN}

Composición y diversidad: A pesar de tener un área casi tres veces menor y una comunicación menos directa con el mar (ver abajo), Hampolol resultó significativamente más diverso que El Remate, en contra de la hipótesis planteada al inicio. Dos explicaciones posibles, una basada en grado de impacto y la otra en factores históricos, se discuten más adelante.

Antes de este estudio, sólo se habían registrado siete especies en los petenes campechanos (Carrillo-Navarro 1995, citado por VillalobosZapata 2004). La ictiofauna conocida en estos ambientes cárstico-palustres se ha incrementado casi cuatro veces. Algunos de los nuevos hallazgos extienden el ámbito de distribución de los taxones en cuestión: en la vertiente del golfo de México, Rivulus tenuis no se había capturado en el estado de Campeche, mientras que Phallichthys fairweatheri, Xiphophorus hellerii y $X$. maculatus no se conocían al norte de Sabancuy $\left(18^{\circ} 58^{\prime} \mathrm{N}\right)$, ni 'Cichlasoma' salvini al norte del río Champotón $\left(19^{\circ} 21^{\prime} \mathrm{N}\right)$ (Miller et al. 2005).

La región de Los Petenes presenta características fisiográficas e hidrológicas similares a las de los Everglades en la Florida, donde Kushlan (1980) observó que las especies de peces se adaptan a las fluctuaciones del nivel del agua, tanto estacionales como interanuales. También en este estudio las condiciones hidrológicas durante las épocas de secas y lluvias determinaron los cambios en las abundancias de las especies presentes en ambos petenes: por ejemplo, Poecilia velifera aumenta su densidad a finales de la época seca. El mismo autor observó además que las densidades más altas se encuentran en cuerpos de agua temporales, con profundidades menores de $0.3 \mathrm{~m}$. Un patrón similar se encontró en los ojos de agua temporales de ambos petenes.

En los petenes estudiados existen cuatro especies de origen marino. Dada la corta distancia a la costa y la eurihalinidad de estos peces, el registro no sería sorprendente, de no ser porque la vía acuática directa al mar presenta, en el caso de Hampolol, obstrucción por azolvamiento, de modo que la comunicación es esporádica y depende de huracanes, lluvias intensas y eventos similares. El ojo de agua principal de Hampolol presentaba 30 años atrás una profundidad de $2 \mathrm{~m}$ (J. Gómez, com. pers.), mientras que en la actualidad no sobrepasa los 0.7 m. En El Remate, la comunicación directa con el mar se mantiene debido a la modificación de un canal de marea natural, lo cual explica la mayor proporción de especies de origen marino en este petén.

Sin embargo, al menos en el caso del sábalo (Megalops atlanticus), se conocen invasiones incluso más notables de localidades dulceacuícolas semiaisladas, por ejemplo cenotes decenas de kilómetros tierra adentro, en Sian Ka'an (Schmitter-Soto et al. 2002).

Relación especie-ambiente: De acuerdo con los resultados del ACC, en el área de estudio se pueden diferenciar dos grupos principales de asociaciones de peces, correlacionadas con las variables ambientales. El primero está 
conformado por las especies que habitan en aguas permanentes y el segundo por las que habitan en aguas temporales. En ambos petenes las asociaciones están influenciadas por la variabilidad en profundidad, oxígeno disuelto, salinidad y temperatura.

Según Kobza et al. (2004), en los Everglades la colonización por peces depende del tamaño y profundidad de los cuerpos de agua, así como la complejidad del hábitat (vegetación acuática y sedimentos alóctonos). Los hábitats más someros $(<0.5 \mathrm{~m})$ se caracterizan por un área mayor y una alta densidad de macrófitas acuáticas, que albergan sobre todo especies de talla pequeña. De igual forma, en Los Petenes los hábitats acuáticos temporales, someros, de fondos lodosos con materia orgánica en descomposición y aguas con poco oxígeno disuelto se diferencian de los permanentes debido a que las especies que los conforman son de talla pequeña (10-40 mm LP), como $R$. tenuis, H. bimaculata, P. fairweatheri, Gambusia spp. y Xiphophorus spp. Estas especies buscan refugio y alimento en estos cuerpos de agua (Schmitter-Soto 1998a).

Asimismo, en los canales se observó una mayor abundancia de vegetación acuática en el fondo, con mayor frecuencia de individuos pequeños de $V$. synspila y $T$. meeki $(\mathrm{LP}<85$ $\mathrm{mm}$ ), quizá por ser lugares con mayor disponibilidad de alimento y protección para los cíclidos (Gamboa-Pérez y Schmitter-Soto 1999).

Se encontraron diferencias significativas en salinidad entre los petenes, siendo El Remate el de mayor salinidad. Esto se verifica en la distribución de las asociaciones de peces, que en El Remate exhibieron mayor afinidad con la salinidad, de acuerdo con el ACC.

En el presente estudio, Gambusia yucatana fue poco frecuente y se encontró en salinidades menores a $2 \%$. De acuerdo con Greenfield et al. (1982), esta especie prefiere ambientes de salinidad relativamente alta. Por otra parte, en este estudio $G$. sexradiata registró una densidad mayor que la de su congénere, mientras que en la vertiente del Caribe, en condiciones de simpatría, G. yucatana tiende a ser más abundante que G. sexradiata (Pérez-León y
Schmitter-Soto 2007). Quizá la explicación radica en que G. yucatana suele preferir las aguas abiertas (Greenfield et al. 1983), hábitat poco disponible en los petenes, a diferencia de las lagunas y cenotes.

Factores ecológicos vs. factores históricos: Diversos estudios han señalado la influencia de las variables ambientales en la composición de las asociaciones de peces (Kushlan 1976, Gelwick et al. 2001, Hoeinghaus et al. 2003, Kobza et al. 2004). Otros autores atribuyen esta influencia no sólo a las variables ambientales, sino también biológicas, como la cobertura de macrófitas, la complejidad del hábitat, las redes tróficas y las relaciones de competencia y depredación (Kushlan 1980, Kupschus y Tremain 2001, Heredia 2002, Petry et al. 2003).

Entre hábitats, en particular entre sitios temporales y permanentes, la distribución de las asociaciones de peces en los petenes se atribuye a variables ambientales (profundidad, salinidad, oxígeno disuelto y temperatura). Entre petenes, el ACC detectó también cierto componente ecológico, que se explica fundamentalmente porque El Remate tiene mayor salinidad y, cabría añadir, una mejor comunicación con el mar. Sin embargo, este factor no contribuye a explicar por qué Hampolol resultó más diverso que El Remate, pues las especies más intolerantes a la salinidad, como Rhamdia guatemalensis, existen en ambos petenes.

Los ambientes palustres en la región de Los Petenes de Campeche deben su origen a diferentes pulsos tectónicos identificables en dos subregiones: norte, de la cual es un sitio representativo el petén El Remate, y sur, en la cual se ubica el petén Hampolol. La primera se caracteriza fisiográficamente por ser una planicie costera subxérica amplia de unos $25 \mathrm{~km}$, y la segunda por ser una planicie costera húmeda de unos $9 \mathrm{~km}$, limitada por los pliegues en bloque de la parte central de Campeche. La subregión sur es geológicamente más antigua que la subregión norte: inició su levantamiento durante el Eoceno, hace 57 millones de años (ma). Posteriormente, en el Oligoceno y Mioceno 
inferior, predominaron ambientes de mares someros en la porción norte, y levantamientos diferenciales más importantes en la porción meridional. Finalmente el levantamiento eustático acumulado determinó un basculamiento sur-norte de los estratos de rocas carbonatadas que componen la región (Emery 1977, LópezRamos 1981, Duch 1988).

Después del levantamiento gradual generalizado, se inició la configuración moderna de la zona costera en una alternancia de transgresiones y regresiones que son más evidentes en la subregión norte. En el Pleistoceno $(80,000$ años), una transgresión interglacial originó cordones litorales, a los cuales se asocia la presencia actual de esteros y lagunas costeras. Enseguida, una regresión ocasionada por una glaciación (18,000 años) provocó un descenso en el nivel del mar de hasta 130 m (Emery 1977, López-Ramos 1981, Duch 1988). Esta última regresión terminó con una gran avance transgresivo, que inundó las geoformas terrestres, marcando el inicio del Holoceno, estabilizando gradualmente, desde hace unos 5000 años, la línea de costa unos 3 ó 4 m por debajo del nivel medio del mar actual.

En esta mayor permanencia geológica de ambientes marinos, se da la adaptación de especies continentales como A. altior, que se caracteriza por su gran abundancia y dominancia en la subregión norte, lo mismo que $P$. velifera. En paralelo, numerosas especies propias de la subprovincia del Petén guatemalteco encuentran su límite norte de distribución hacia la latitud de los petenes. Muchos extensiones de ámbito descubiertas en este estudio se refieren a especies, como los Xiphophorus, Phallichthys y 'Cichlasoma' salvini, que están ausentes en el norte de la península. Es interesante que algunas especies sureñas, como $A$. aeneus, Petenia splendida y $X$. hellerii, se hayan registrado en el petén Hampolol, mas no en El Remate, como si la frontera biogeográfica (que en realidad es más bien una zona de transición faunística) se ubicara justo entre ambas localidades.

En relación con el género Astyanax, Schmitter-Soto (1998b) reportó la distribución de varias poblaciones en probable introgresión (individuos intermedios entre $A$. aeneus y $A$. altior) en la meseta de Zohlaguna, la laguna X-Hohomil y los cenotes de Tulum, en Quintana Roo. Posiblemente, esta misma introgresión se encuentre en el petén Hampolol, en donde la abundancia del posible híbrido $A$. aeneus $\times$ altior es alta en comparación con las de $A$. altior y A. aeneus. Apenas unos kilómetros al norte, en El Remate, la única especie de Astyanax presente es $A$. altior.

Las diferencias en composición y diversidad entre ambos petenes pueden entonces explicarse -al margen de los invasores marinos que pudieron penetrar con mayor facilidad en El Remate- por razones históricas: especies meridionales que encuentran su límite de distribución en Hampolol y especies septentrionales que, si bien pueden encontrarse esporádicamente al sur de Los Petenes (A. altior en Calakmul [Vega-Cendejas y Hernández 2004], $P$. velifera en la laguna de Términos [Miller et al. 2005]), son mucho más abundantes y frecuentes en la parte norte de la península de Yucatán, sin que ello pueda entenderse por alguna preferencia ecológica, sino más bien en términos de biogeografía histórica (SchmitterSoto 1999, 2002).

Impacto antropogénico: La presencia de especies exóticas (las tilapias Oreochromis aureus y $O$. niloticus) en el petén Hampolol se debe a su introducción con fines acuiculturales en áreas cercanas y a su dispersión a través de una inundación generalizada a causa de las lluvias torrenciales generadas por el huracán Roxana en octubre de 1995 (J. Gómez, com. pers.). Lo anterior ha generado un impacto ecológico en la reserva que hasta el momento no ha sido evaluado, y que puede causar una variación en la composición de especies de la comunidad, desplazando o incluso induciendo la extinción de especies nativas (MartínezPalacios y Ross 1994, Schmitter-Soto y Caro 1997). Será importante dar seguimiento a la composición y diversidad de este petén.

Al margen de la introducción de especies exóticas, ambos petenes han sido perturbados por actividades humanas. Esto es más notorio 
en el caso de El Remate, por ser un balneario popular. Además de las alteraciones propias del acondicionamiento con fines recreativos, El Remate presenta un impacto en su dinámica hidrológica, generado por la construcción de la carretera Tankuché-Isla Arena y la modificación del canal de marea natural que vincula los ojos de agua con el mar.

El petén Hampolol, en cambio, está rodeado por una vegetación arbórea que aún presenta un buen estado de conservación. En el caso de El Remate, como se comentó, es un sistema con un fuerte impacto por su uso recreativo, lo que ha deteriorado la vegetación arbórea circundante.

Debido al estado de deterioro actual de ambos petenes, estas áreas requieren de un plan de manejo y restauración adecuados para evitar la pérdida de refugios acuáticos para los peces, como ha sucedido en la región de los Everglades (Kobza et al. 2004).

\section{AGRADECIMIENTOS}

El presente trabajo fue parte de la tesis de maestría de la primera autora. Se agradece a Mirella Hernández de Santillana y a Alfonso González Díaz la ayuda en la identificación de especies en el laboratorio, a Roberto Herrera Pavón y Alex Acosta su colaboración en el campo y a Jorge Navarro la asesoría en estadística. También se agradece a la Universidad Autónoma de Campeche (Centro EPOMEX y CEDESU) y al ejido Tankuché por brindar las facilidades necesarias para realizar este trabajo, y a la SEMARNAT por el permiso de recolecta del material (No. DGOPA/03981/150305/.-2128).

\section{RESUMEN}

Los petenes son pequeños manantiales y arroyos asociados que drenan a sistemas palustres en áreas cársticas costeras. Se estudió la composición, distribución y abundancia de la ictiofauna en dos petenes del noroeste de Campeche, en el manantial principal, el arroyo asociado, y pequeños ojos de agua secundarios (temporales), en dos épocas del año. Se registraron variables ambientales y tipos de impacto antrópico en cada petén. Se encontraron en total 27 especies de peces, con extensiones de ámbito de 'Cichlasoma' salvini, Rivulus tenuis, Phallichthys fairweatheri, Xiphophorus hellerii y X. maculatus. La especie dominante en ambas épocas fue Astyanax (probables híbridos A. aeneus $\times$ altior en Hampolol, A. altior en El Remate). Hubo diferencia significativa en diversidad entre ambos petenes. El análisis de conglomerados diferenció dos agrupaciones dentro de cada petén: aguas permanentes y sitios temporales. Las variables ambientales (excepto salinidad) presentaron diferencias significativas por sitio y época; un análisis de correspondencia canónica indicó que la distribución de los conjuntos de peces tuvo influencia de las variables ambientales en ambas temporadas. En términos de composición, los factores históricos juegan un papel en las diferencias ictiológicas entre ambos petenes, sobre todo por lo que concierne a la presencia de híbridos de Astyanax, así como Xiphophorus hellerii en el petén del sur (Hampolol) y de Poecilia velifera en el petén del norte (El Remate).

Palabras clave: composición de especies, impacto antropogénico, asociaciones de peces, petén, Campeche, península de Yucatán.

\section{REFERENCIAS}

Bloom, S.A. 1981. Similarity indices in community studies: potential pitfalls. Mar. Ecol. Progr. Ser. 5: 125-128.

de la Cruz, G. 1994. ANACOM. Sistema para el análisis de comunidades. Manual del usuario. Centro de Investigación y Estudios Avanzados, Mérida, Yucatán, México.

Diario Oficial de la Federación (DOF). 1999. Decreto por el que se declara área natural protegida, con el carácter de reserva de la biosfera, la región del Estado de Campeche conocida como Los Petenes, con una superficie total de 282,857-62-70.6 hectáreas. Diario Oficial de la Federación, 24 de mayo de 1999, México D.F.

Duch, J. 1988. La conformación territorial del estado de Yucatán: componentes del medio físico. Universidad Autónoma de Chapingo, Centro Regional de la Península de Yucatán, Mérida, Yucatán, México.

Emery, K.O. 1977. The continental shelves, p. 33-44. In: Menard, H. W. (Eds.). Ocean Sciences. W.H. Freeman, San Francisco, California, EEUU.

Gamboa-Pérez, H.C. \& J.J Schmitter-Soto. 1999. Distribution of cichlid fishes in the littoral of Lake Bacalar, Yucatan Peninsula. Env. Biol. Fishes 54: $35-43$.

Gelwick, F.P., S. Akin, D. Albrey \& K.O. Winemiller. 2001. Fish assemblage structure in relation to environmental 
variation in a Texas Gulf coastal wetland. Estuaries 24: $285-296$

Greenfield, D.W. \& J.E. Thomerson 1997. Fishes of the continental waters of Belize. University of Florida, Gainesville, Florida, EEUU.

Greenfield, D.W., T.A. Greenfield \& S.L. Brinton 1983. Spatial and trophic interaction between Gambusia sexradiata and Gambusia puncticulata yucatana (Pisces: Poeciliidae) in Belize, Central America. Copeia 1983: 598-607.

Greenfield, D.W., T.A. Greenfield \& D. M. Wildrick. 1982. The taxonomy and distribution of the species of Gambusia (Pisces: Poeciliidae) in Belize, Central America. Copeia 1982: 128-147.

Heredia, M. 2002. Contribución al conocimiento taxonómico y ecológico de la familia Cichlidae: Ictiofauna de la Reserva de la Biósfera de Calakmul, Campeche. Tesis de Licenciatura, Universidad Autónoma Metropolitana, México, D.F., México.

Hoeinghaus, D.J., C. A. Layman, D. Albrey \& K.O Winemiller. 2003. Spatiotemporal variation in fish assemblage structure in tropical floodplain creeks. Env. Biol. Fishes 67: 379-387.

Kobza, R.M., J.C. Trexler, W.F. Loftus \& S.A. Perry. 2004 Community structure of fishes inhabiting aquatic refuges in a threatened karst wetland and its implications for ecosystem management. Biol. Conserv. 116 $153-165$

Kupschus, S. \& D. Tremain. 2001. Associations between fish assemblages and environmental factors in nearshore habitats of a subtropical estuary. J. Fish Biol. 58: 1383-1403.

Kushlan, J.A. 1976. Environmental stability and fish community diversity. Ecology 57: 821-825.

Kushlan, J.A. 1980. Population fluctuations of Everglades fishes. Copeia 1980: 870-874.

Loftus, W.F. \& J.A. Kushlan. 1987. Freshwater fishes of Southern Florida. Bull. Florida St. Mus. Biol. Sci. 31: 1-344.

López-Ramos, E. 1981. Carta geológica de la península de Yucatán. Esc. 1:500.000. Instituto de Geología, UNAM, México, D.F., México.

Lowe-McConnell, R.M. 1987. Ecological studies in tropical fish communities. Cambridge University, Cambridge, Reino Unido.

Lyons, J., S. Navarro-Pérez, P.A. Cochran, E Santana C. \& M Guzmán-Arroyo. 1995. Index of biotic integrity based on fish assemblages for the conservation of streams and rivers in west-central Mexico. Conserv. Biol. 9: 569-584.

Martínez-Palacios, C.A. \& L.G. Ross. 1994. Biología y cultivo de la mojarra latinoamericana Cichlasoma urophthalmus. CONACYT, México, D.F., México.

Mas, J.F. \& J. Correa. 2000. Análisis de la fragmentación del paisaje en el área protegida "Los Petenes", Campeche, México. Invest. Geogr., Bol. Inst. Geogr. UNAM 43: 43-59.

Maya Martínez, F. 1998. Distribución y abundancia de peces continentales en la Reserva de la Biósfera de Sian Ka' an, Quintana Roo. Tesis de Licenciatura, Universidad Michoacana de San Nicolás de Hidalgo, Morelia, Michoacán, México.

Miller, R.R., W.L. Minckley \& S.M. Norris. 2005. Freshwater fishes of México. University of Chicago, Chicago, Illinois, EEUU.

Nelson, J.S. 1994. Fishes of the World. $3^{\text {a }}$ Ed. J. Wiley, Nueva York, Nueva York, EEUU.

Pérez-León, S. \& J.J. Schmitter-Soto. 2007. Distribución y taxonomía del género Gambusia (Teleostei: Poeciliidae) en el norte y oriente de la península de Yucatán, México. Univ. Cienc., Univ. Juárez Autón. Tabasco 23: 167-171.

Petry, P., P.B. Bayley \& D.F. Markle. 2003. Relationships between fish assemblages, macrophytes and environmental gradients in the Amazon River floodplain. J. Fish Biol. 63: 547-579.

Schmitter-Soto, J.J. 1998a. Catálogo de los peces continentales de Quintana Roo. Guías científicas ECOSUR. El Colegio de la Frontera Sur, San Cristóbal de Las Casas, Chiapas, México.

Schmitter-Soto, J.J. 1998b. Diagnosis of Astyanax altior (Characidae), with a morphometric analysis of Astyanax in the Yucatan Peninsula. Ichthyol. Explor. Freshwat. 8(4): 349-358.

Schmitter-Soto, J.J. 1999. Distribution of continental fishes in northern Quintana Roo, Mexico. Southw. Nat. 44: 166-172.

Schmitter-Soto, J.J. 2002. Ictiogeografía de Yucatán, p. 103-116. In: M.L. Lozano-Vilano (Ed.), Libro jubilar en honor al Doctor Salvador Contreras Balderas. Universidad Autónoma de Nuevo León, Monterrey, Nuevo León, México. 
Schmitter-Soto, J.J. \& C.I. Caro. 1997. Distribution of tilapia, Oreochromis mossambicus (Perciformes: Cichlidae), and water body characteristics in Quintana Roo, Mexico. Rev. Biol. Trop. 45: 1257-1262.

Schmitter-Soto, J.J \& H.C. Gamboa-Pérez. 1996. Distribución de peces continentales en el sur de Quintana Roo, México. Rev. Biol. Trop. 44: 199-212.

Schmitter-Soto, J.J., A.M. Arce-Ibarra \& L. VásquezYeomans. 2002. Records of Megalops atlanticus in the Mexican Caribbean coast. Contrib. Mar. Sci. 35: 34-42.

Underwood, A. J. 1997. Experiments in ecology. Their logical design and interpretation using analysis of variance. Cambridge University, Cambridge, Reino Unido.
Vega-Cendejas, M.E. y M. Hernández de Santillana. 2004. Los peces de la Reserva de la Biósfera de Calakmul, Campeche. Conservation International/CINVESTAV, Mérida, Yucatán, México.

Villalobos-Zapata, G.J. 2004. Reservas de la biosfera costeras: Los Petenes y Ría Celestún, cap. 27, p. $397-$ 411. In E. Rivera Arriaga, G.J. Villalobos Zapata, I. Azuz Adeath \& F. Rosado May (Eds.), El manejo costero en México. Universidad Autónoma de Campeche / SEMARNAT / CETYS-Universidad, Universidad de Quintana Roo, Campeche, Campeche, México.

Yáñez-Arancibia, A. y 23 coautores. 1996. Caracterización ecológica ambiental y de los recursos naturales de la región de Los Petenes en Campeche. Periódico Oficial del Gobierno Constitucional del Estado de Campeche, Segunda Sección 5(1198): 1- 426. 
